Graziella Bonetti*, Filippo Manelli, Andrea Patroni, Alessandra Bettinardi, Gianluca Borrelli, Gianfranco Fiordalisi, Antonio Marino, Annamaria Menolfi, Sara Saggini, Roberta Volpi, Adriano Anesi and Giuseppe Lippi

\title{
Laboratory predictors of death from coronavirus disease 2019 (COVID-19) in the area of Valcamonica, Italy
}

https://doi.org/10.1515/cclm-2020-0459

Received April 6, 2020; accepted April 13, 2020; previously published online April 28, 2020

\section{Abstract}

Background: Comprehensive information has been published on laboratory tests which may predict worse outcome in Asian populations with coronavirus disease 2019 (COVID-19). The aim of this study is to describe laboratory findings in a group of Italian COVID-19 patients in the area of Valcamonica, and correlate abnormalities with disease severity.

Methods: The final study population consisted of 144 patients diagnosed with COVID-19 (70 who died during hospital stay and 74 who survived and could be discharged) between March 1 and 30, 2020, in Valcamonica Hospital. Demographical, clinical and laboratory data were collected upon hospital admission and were then correlated with outcome (i.e. in-hospital death vs. discharge).

Results: Compared to patients who could be finally discharged, those who died during hospital stay displayed significantly higher values of serum glucose, aspartate aminotransferase (AST), creatine kinase (CK), lactate dehydrogenase (LDH), urea, creatinine, high-sensitivity cardiac troponin I (hscTnI), prothrombin time/ international normalized ratio (PT/INR), activated partial

*Corresponding author: Graziella Bonetti, MD, Laboratory of Clinical Pathology, ASST-Valcamonica, Via A. Manzoni, 142, 25040 Esine, Brescia, Italy, E-mail: graziella.bonetti@asst-valcamonica.it Filippo Manelli: Emergency Department, ASST-Valcamonica, Esine, Brescia, Italy

Andrea Patroni: Hospital Infections Committee, ASST-Valcamonica, Esine, Brescia, Italy

Alessandra Bettinardi, Gianluca Borrelli, Gianfranco Fiordalisi, Antonio Marino, Annamaria Menolfi, Sara Saggini and Roberta Volpi: Laboratory of Clinical Pathology, ASST-Valcamonica, Esine, Brescia, Italy

Adriano Anesi: Laboratory of Clinical Pathology, Maggiore Hospital of Lodi, Lodi, Italy

Giuseppe Lippi: Section of Clinical Biochemistry, University of Verona, Verona, Italy thromboplastin time (APTT), D-dimer, $\mathrm{C}$ reactive protein (CRP), ferritin and leukocytes (especially neutrophils), whilst values of albumin, hemoglobin and lymphocytes were significantly decreased. In multiple regression analysis, LDH, CRP, neutrophils, lymphocytes, albumin, APTT and age remained significant predictors of in-hospital death. A regression model incorporating these variables explained $80 \%$ of overall variance of in-hospital death.

Conclusions: The most important laboratory abnormalities described here in a subset of European COVID-19 patients residing in Valcamonica are highly predictive of in-hospital death and may be useful for guiding risk assessment and clinical decision-making.

Keywords: coronavirus disease 2019; COVID-19; laboratory medicine; laboratory tests.

\section{Introduction}

Coronaviruses are enveloped, non-segmented, positivesense RNA viruses, known to cause disease in humans and animals, ranging from common cold to more severe and even deadly respiratory infections [1]. Since 2003, two highly pathogenic human betacoronaviruses have emerged in two separate outbreaks, i.e. severe acute respiratory syndrome coronavirus (SARS-CoV-1) and Middle East respiratory syndrome coronavirus (MERS-CoV-1) $[2,3]$. These microorganisms have caused the homologous diseases SARS and MERS, characterized by lower respiratory tract infection (i.e. pneumonia) and extra-pulmonary manifestations, affecting many people and causing several deaths in their regions of emergence.

In December 2019, a new strain of coronavirus, finally called severe acute respiratory syndrome coronavirus 2 (SARS-CoV-2) due to its high sequence identity with SARS-CoV-1, was first isolated from patients developing an acute respiratory syndrome in Wuhan, China [4], and the ensuing pathology has been defined as coronavirus disease 2019 (COVID-19) [5, 6]. At the time of writing this article, the last statistics of the World Health Organization (WHO) revealed that COVID-19 had already affected over 
1.2 million people from almost every country worldwide, causing as many as 70,000 deaths [7]. Italy is one of the most deeply involved country, for the number of cases (over 120,000), deaths (over 15,000) and especially the mortality rate, which is now as high as $12 \%$. Beside this sizeable death rate, the clinical course of this pathology may be complicated in up to $15 \%$ by the onset of a severe form of intestinal pneumonia, which may then evolve into acute respiratory distress syndrome (ARDS), viremia and viral sepsis, intravascular coagulopathies, targeted or multiple organ failure (MOF), up to death $[6,8]$.

Although the clinical characteristics of COVID-19 have been already straightforwardly defined in China and abroad $[9,10]$, studies on the leading laboratory abnormalities developing in patients with COVID-19 outside the Asian region, and especially in Europe, are currently lacking to the best of our knowledge. This gap shall be urgently filled, whereby the key role of laboratory diagnostics in diagnosing, but also in prognosticating and monitoring, COVID-19 is now well recognized [11-13].

A recent systematic literature review highlighted the most important abnormalities that have been observed in Asian COVID-19 patients [14]. Briefly, lymphopenia, along with increased values of $\mathrm{C}$ reactive protein (CRP), lactate dehydrogenase (LDH), erythrocyte sedimentation rate (ESR) and D-dimer, was found to be commonplace in these patients. Some other laboratory parameters were found to predict progression into severe or even critical forms of COVID-19, including neutrophilia, leukocytosis, lymphopenia, increased values of total bilirubin, LDH, alanine aminotransferase (ALT), aspartate aminotransferase (AST), creatinine, cardiac biomarkers, prothrombin time (PT), D-dimer, CRP and procalcitonin. Abnormalities of hemostasis tests [15], cardiac biomarkers [16] and procalcitonin [17] have then been convincingly confirmed in subsequent studies.

Therefore, the aim of the present study was to investigate and describe the leading laboratory findings in a group of Italian COVID-19 patients in the area of Valcamonica, and correlate these abnormalities with disease severity (i.e. death).

\section{Materials and methods}

\section{Patients}

From an initial group of 518 COVID-19 patients admitted to the Emergency Department of the Valcamonica Hospital (Esine, Brescia, Lombardy, Italy) and diagnosed with COVID-19 between March 1 and 30, 2020, two different cohorts from those hospitalized $(n=230)$ were selected for having complete clinical and laboratory data. The first cohort included 74 COVID-19 patients who survived and could hence be discharged, whilst the second group encompassed 70 patients who died within their hospital stay. All patients were diagnosed with COVID-19 according to current standards, i.e. displaying suggestive findings at chest computed tomography (CT; the classic ground glass pattern of interstitial pneumonia for a minimum of 35\%-40\% of lung parenchyma) and positive results of real-time reverse transcriptase polymerase chain reaction (RT-PCR) for SARS-CoV-2.

\section{Data collection}

Clinical history, signs, symptoms and results of laboratory investigations at hospital admission were collected from a standard form used for reporting data on infectious diseases to the Italian Ministry of Health, from the local Laboratory Information System (LIS), as well as from medical records. All clinical and laboratory data described in this article have been recorded upon hospital admission.

Routine hematological testing, including hemoglobin $(\mathrm{Hb})$ concentration, white blood cell (WBC), platelets (PLT), neutrophil and lymphocytes counts, was carried out in blood samples anticoagulated with tripotassium ethylenediaminetetraacetic acid (K-EDTA; Becton Dickinson, Franklin Lakes, NJ, USA) on Sysmex XN instrumentation and using proprietary reagents (Sysmex Corporation, Kobe, Japan). Coagulation tests, including activated partial thromboplastin time (APTT), prothrombin time/international normalized ratio (PT/INR) and D-dimer were carried out on plasma collected into 3.2\% buffered sodium citrate blood tubes (Becton Dickinson, Franklin Lakes, NJ, USA), using Stago coagulation analyzers STA compact Max 3 (Stago, Asnières-sur-Seine, France) and proprietary reagents. Clinical chemistry and immunochemical testing were performed on lithium heparin plasma samples (Becton Dickinson, Franklin Lakes, NJ, USA) using Abbott Alinity Systems (Abbott, Chicago, IL, USA), and included measurement of serum glucose, urea, creatinine, sodium, potassium, chloride, AST, ALT, CK, LDH, $\gamma$-glutamyltransferase (GGT), total bilirubin, albumin, ferritin, CRP and high-sensitive cardiac troponin I (hscTnI). An identical set of analyzers was used throughout the study period. The quality of results was validated with internal quality control (IQC) procedures and participation to an External Quality Assessment Scheme (EQAS). Clinical and laboratory information was collected during clinical workout and the study was preliminary approved by the Ethical Committee of Brescia (certificate no. NP 4036). The study was carried out in accordance with the Declaration of Helsinki and with the term of the local legislation.

\section{Statistical analysis}

Data distribution was explored with the Kolmogorov-Smirnov test. As normal distribution could not be confirmed, the Mann-Whitney test for independent samples was used for comparing laboratory parameters between COVID-19 patients who survived and those who died. Data were expressed as median and interquartile range (IQR). Proportions for categorical variables were compared with the chi squared $\left(\chi^{2}\right)$ test. Multiple regression, with the backward method, was used to analyze the relationship between outcome (in-hospital death or discharge) and laboratory parameters at hospital admission, to identify possible predictors of in-hospital death. Statistical 
analysis was performed using the MedCalc software, version 19.2 (Ostend, Belgium). Values of $\mathrm{p}<0.05$ were considered statistically significant.

\section{Results}

\section{Clinical characteristics}

The clinical characteristics of the entire group of COVID-19 patients included in this study are shown in Table 1 . The population of COVID-19 patients who died was basically older than that of survivors. A marginally higher prevalence of chronic respiratory disorders in COVID-19 patients who died during hospital stay than in those who survived was the only other significant difference found in our cohort of COVID-19 patients (20.5\% vs. $8.0 \%$; $\mathrm{p}=0.031$ ), as no other significant differences could be found in demographical and clinical variables (Table 1).

The complete laboratory data of the two cohorts of COVID-19 patients who died or survived are shown in Table 2. Compared with patients who could be discharged, those who died during hospital stay exhibited significantly higher values of glucose, AST, CK, LDH, urea, creatinine, hscTnI, PT/INR, APTT, D-dimer, CRP, ferritin, WBC and neutrophils, whilst the values of albumin, hemoglobin and lymphocytes were found to be significantly decreased. In multiple regression analysis, where in-hospital death was entered as the dependent variable and clinical and laboratory parameters associated with mortality in univariate analysis as independent variables, LDH $(p<0.001)$, CRP $(p=0.045)$, neutrophils $(p=0.010)$, lymphocytes $(p=0.003)$, albumin $(p=0.003)$, WBC $(p=0.016)$, APTT $(p=0.007)$ and age $(p<0.001)$ remained significant predictors. A regression model incorporating these variables contributed to explain $80 \%$ of the overall variance of in-hospital death.

\section{Discussion}

The identification of reliable clinical and laboratory predictors, which may help identify a subset of COVID-19 patients at enhanced risk of developing severe forms of disease (e.g. needing ventilation support and/or intensive care, or even dying), is foremost for guiding the managed care, especially in healthcare settings whose resources have been overwhelmed by the huge number of COVID-19 cases needing sub-intensive or intensive care.

In keeping with this compelling need, the results of our investigation provide a concise description of the leading clinical and laboratory abnormalities characterizing a subset of COVID-19 patients with unfavorable outcome (i.e. death). In agreement with the results of a recent metaanalysis, which exclusively included Asian population data [18], we observed that a number of laboratory tests exhibit

Table 1: Clinical and demographical characteristics of coronavirus disease 2019 (COVID-19) patients who died during hospital stay and those who survived and could be discharged.

\begin{tabular}{|c|c|c|c|}
\hline Clinical and demographical characteristics & $\begin{array}{r}\text { In-hospital death } \\
(\mathrm{n}=70)\end{array}$ & $\begin{array}{r}\text { Discharged } \\
(n=74)\end{array}$ & p-Value \\
\hline Age, years & $78.0(64.2-84.0)$ & $62.1(53.0-72.8)$ & $<0.001$ \\
\hline Males, \% & $45(64.3 \%)$ & $51(68.9 \%)$ & 0.383 \\
\hline Females, \% & $25(35.7 \%)$ & $24(31.1 \%)$ & \\
\hline Days from symptoms onset to hospital admission & 4 & 7 & \\
\hline Days from hospital admission to outcome & 6 & 2 & \\
\hline Chronic diseases & 49 (70\%) & $43(57.3 \%)$ & 0.114 \\
\hline Cancer & $9(12.9 \%)$ & $6(8.0 \%)$ & 0.335 \\
\hline Diabetes & $21(30 \%)$ & $16(21.3 \%)$ & 0.231 \\
\hline Cardiovascular disease & $38(54.3 \%)$ & $33(44.0 \%)$ & 0.217 \\
\hline Immunodeficiencies & $2(2.8 \%)$ & $0(0.0 \%)$ & 0.146 \\
\hline Chronic respiratory diseases & $14(20.5 \%)$ & $6(8.0 \%)$ & 0.031 \\
\hline Chronic kidney diseases & $9(12.9 \%)$ & $3(4.0 \%)$ & 0.053 \\
\hline Metabolic diseases & $10(14.3 \%)$ & $7(9.3 \%)$ & 0.351 \\
\hline BMI 30-40 & $11(15.7 \%)$ & $5(6.7 \%)$ & 0.085 \\
\hline $\mathrm{BMI}>40$ & 1 (1.4\%) & $0(0.0 \%)$ & 0.306 \\
\hline
\end{tabular}

Results are shown as median and interquartile range (between brackets). BMI, body mass index. 
Table 2: Comparison of laboratory parameters between coronavirus disease 2019 (COVID-19) patients who died during hospital stay and those who survived and could be discharged.

\begin{tabular}{lrrr}
\hline Parameters & In-hospital death $(\mathbf{n}=70)$ & Discharged $(\mathbf{n}=74)$ & p-Value \\
\hline Glucose, mmol/L & $7.63(6.44-10.60)$ & $6.10(5.61-6.77)$ & $<0.001$ \\
Albumin, g/L & $34.6(31.8-35.9)$ & $36.5(33.8-39.3)$ & $<0.001$ \\
ALT, U/L & $34(23-49)$ & $31.5(21-46)$ & 0.516 \\
AST, U/L & $61.5(44-83)$ & $45(25-59)$ & 0.001 \\
Bilirubin total, $\mu \mathrm{mol} / \mathrm{L}$ & $11.7(8.7-19.5)$ & $10.6(8.7-13.9)$ & 0.109 \\
GGT, U/L & $56(31.25-105.0)$ & $45(28.0-86.75)$ & 0.232 \\
Lipase, U/L & $33(25.0-41.25)$ & $38(31.0-45.0)$ & 0.013 \\
CK, U/L & $168(92.0-350.5)$ & $92.5(60.0-198.0)$ & 0.001 \\
LDH, U/L & $521(416-636)$ & $316(247-397.25)$ & $<0.001$ \\
Sodium, mmol/L & $138(135-141)$ & $137(135-140)$ & 0.655 \\
Potassium, mmol/L & $4.0(3.6-4.55)$ & $3.9(3.45-4.20)$ & 0.165 \\
Chloride, mmol/L & $101(98.5-103.5)$ & $101(98.0-104.0)$ & 0.722 \\
Urea, $\mathrm{mmol} / \mathrm{L}$ & $23.4(16.1-33.6)$ & $12.1(10.4-19.3)$ & $<0.001$ \\
Creatinine, $\mu \mathrm{mol} / \mathrm{L}$ & $124(85-155)$ & $91(73-104)$ & $<0.001$ \\
hscTnl, $\mathrm{ng} / \mathrm{L}$ & $47.5(2.0-290.0)$ & $8.0(5.1-15.0)$ & $<0.001$ \\
PT/INR & $1.12(1.03-1.28)$ & $1.04(1.00-1.10)$ & 0.001 \\
APTT, s & $34(29-37)$ & $31(29-34)$ & 0.014 \\
D-dimer, g/L & $1.99(1.27-3.63)$ & $0.91(0.60-2.03)$ & $<0.001$ \\
CRP, g/L & $165.65(82.45-241.4)$ & $60.3(23.6-154.4)$ & $<0.001$ \\
Ferritin, $\mathrm{ng} / \mathrm{mL}$ & $1285(431-2409)$ & $701.5(382-1475)$ & 0.01 \\
Hb, $\mathrm{g} / \mathrm{L}$ & $130(114-144)$ & $137(125-150)$ & 0.024 \\
WBC, $\times 10^{9} / \mathrm{L}$ & $6.64(5.63-12.16)$ & $5.605(4.38-7.60)$ & 0.002 \\
Neutrophils, $\times 10^{9} / \mathrm{L}$ & $5.59(4.45-9.72)$ & $4.13(2.72-5.79)$ & $<0.001$ \\
Lymphocytes, $\times 10^{9} / \mathrm{L}$ & $0.75(0.56-1.01)$ & $1.04(0.81-1.30)$ & $<0.001$ \\
Platelets, $\times 10^{9} / \mathrm{L}$ & $178(132-216)$ & $189(148-230)$ & 0.237 \\
\hline
\end{tabular}

Results are shown as median and interquartile range (between brackets). The parameters were compared using the Mann-Whitney test for independent samples. APTT, activated partial thromboplastin time; AST, aspartate aminotransferase; ALT, alanine aminotransferase; CK, creatine kinase; CRP, C reactive protein; GGT, gamma-glutamyltransferase; Hb, hemoglobin; hscTnl, high-sensitive cardiac troponin I; LDH, lactate dehydrogenase; PT/INR, prothrombin time/international normalized ratio; WBC, white blood cells.

significant abnormalities in COVID-19 Italian patients who died compared to those who survived. This is the very first report of this type in a European population to the best of our knowledge. Even more importantly, we could also demonstrate that some of these specific parameters (i.e. older age, increased values of LDH, CRP, neutrophils and APTT, and decreased values of lymphocytes and albumin) were found to be useful independent predictors of worse outcome (i.e. death), so their combined assessment would contribute to distinguish patients experiencing in-hospital death from those who could be discharged with an accuracy as high as $80 \%$. The incorporation of these variables within clinical and laboratory algorithms may hence be seen as a valuable perspective for targeting, by means of more aggressive therapeutic interventions or narrow monitoring, patients at risk of developing severe and/or critical disease within an already overwhelmed, or even deranged, healthcare environment.

In detail, the observation of hematological abnormalities, especially concerning decreased lymphocyte count and increased neutrophil count, is reasonably explainable. It has been recently demonstrated that lymphocytes actively express the angiotensin converting enzyme 2 (ACE2), which is the leading SARS-CoV-2 receptor at cell surface [19]. It is hence quite reasonable that these cells would be injured by the virus, and that their gradual decline into the circulation may be associated with lymphocytic dysfunction and immunosuppression, finally contributing to expose patients to a higher risk of co-infections and worse prognosis, as also described elsewhere [20]. Directly connected to this important aspect is the finding that neutrophil count and CRP values were found to be higher in our COVID-19 patients who died than in those who survived. Both these laboratory parameters reflect the possible presence of viremia, and especially of bacterial co-infection [21], which can frequently complicate the clinical course of COVID-19, thus representing a well-known unfavorable prognostic factor [22]. The higher values of LDH found in COVID-19 patients who died than in those who survived is neither 
surprising. Reliable evidence has been provided that increased LDH values reflect the extent of lung injury in patients with ARDS, including those with the previous, and relatively similar, coronavirus disease SARS [23]. The significant association between APTT and in-hospital death deserves a final consideration. Severe hemostasis derangements, mostly reflective of intravascular coagulopathies and/or DIC, have been described in as many as $71 \%$ of patients dying of COVID-19 [8]. This evidence is mirrored by the findings of our study, whereby we also observed increased values of D-dimer, PT/INR and especially of APTT in the subset of our COVID-19 patients who died compared to those who survived. It can hence be reasonably speculated that hemostasis disturbances, mostly reflecting the presence of intravascular or consumption coagulopathies, may be frequent in COVID-19 patients with more severe forms of disease, and may then substantially contribute to enhance their risk of death [24].

In conclusion, we have described here for the first time the most characteristic laboratory abnormalities which may predict unfavorable clinical outcomes (i.e. in-hospital death) in a subset of European COVID19 patients residing in Valcamonica. These laboratory tests, which are highly predictive of in-hospital death, may be useful for guiding risk assessment and clinical decision-making.

Research funding: None declared.

Author contributions: All authors have accepted responsibility for the entire content of this manuscript and approved its submission.

Competing interests: Authors state no conflict of interest. Informed consent: Informed consent was obtained from all individuals included in this study.

Ethical approval: Clinical and laboratory information was collected during clinical workout and the study was preliminary approved by the Ethical Committee of Brescia (certificate no. NP 4036). The study was carried out in accordance with the Declaration of Helsinki and with the term of the local legislation.

\section{References}

1. Richman DD, Whitley RJ, Hayden FG, editors. Clinical virology, 4th ed. Washington: ASM Press, 2016.

2. Ksiazek TG, Erdman D, Goldsmith CS, Zaki SR, Peret T, Emery S, et al. A novel coronavirus associated with severe acute respiratory syndrome. N Engl J Med 2003;348:1953-66.
3. de Groot RJ, Baker SC, Baric RS, Brown CS, Drosten C, Enjuanes L, et al. Middle East respiratory syndrome coronavirus (MERS-CoV): announcement of the Coronavirus Study Group. J Virol 2013;87:7790-92.

4. Chan JF, Yuan S, Kok KH, To KK, Chu H, Yang J, et al. A familial cluster of pneumonia associated with the 2019 novel coronavirus indicating person-to person transmission: a study of a family cluster. Lancet 2020;395:514-23.

5. Guan W-J, Ni Z-Y, Hu Y, Liang WH, Ou CQ, He JX, et al. Clinical characteristics of 2019 novel coronavirus infection in China. N Engl J Med 2020. doi: 10.1056/NEJMoa2002032 [Epub ahead of print].

6. Huang C, Wang Y, Li X, Ren L, Zhao J, Hu Y, et al. Clinical features of patients infected with 2019 novel coronavirus in Wuhan, China. Lancet 2020;395:497-506.

7. World Health Organization. Coronavirus disease (COVID-19) outbreak situation https://www.who.int/emergencies/diseases/ novel-coronavirus-2019. Accessed: 5 April 2020.

8. Tang N, Li D, Wang X, Sun Z. Abnormal Coagulation parameters are associated with poor prognosis in patients with novel coronavirus pneumonia. J Thromb Haemost 2020;18:844-7.

9. Mattiuzzi C, Lippi G. Which lessons shall we learn from the 2019 novel coronavirus outbreak? Ann Transl Med 2020;8:48.

10. Wu Z, McGoogan JM. Characteristics of and important lessons from the coronavirus disease 2019 (COVID-19) outbreak in China: summary of a report of 72314 cases from the Chinese center for disease control and prevention. J Am Med Assoc 2020. doi: 10.1001/jama.2020.2648 [Epub ahead of print].

11. Lippi G, Plebani M. The critical role of laboratory medicine during coronavirus disease 2019 (COVID-19) and other viral outbreaks. Clin Chem Lab Med 2020;58:1063-9.

12. Lippi G, Plebani M. The novel coronavirus (2019-nCoV) outbreak: think the unthinkable and be prepared to face the challenge. Diagnosis (Berl) 2020;7:79-81.

13. Lippi G, Simundic AM, Plebani M. Potential preanalytical and analytical vulnerabilities in the laboratory diagnosis of coronavirus disease 2019 (COVID-19). Clin Chem Lab Med 2020;58:1070-6.

14. Lippi G, Plebani M. Laboratory abnormalities in patients with COVID-2019 infection. Clin Chem Lab Med 2020;58:1131-4.

15. Han H, Yang L, Liu R, Liu F, Wu K, Li J, et al. Prominent changes in blood coagulation of patients with SARS-CoV-2 infection. Clin Chem Lab Med 2020;58:1116-20.

16. Lippi G, Lavie CJ, Sanchis-Gomar F. Cardiac troponin I in patients with coronavirus disease 2019 (COVID-19): evidence from a metaanalysis. Prog Cardiovasc Dis 2020. pii: S0033-0620(20)300554. doi: 10.1016/j.pcad.2020.03.001 [Epub ahead of print].

17. Lippi G, Plebani M. Procalcitonin in patients with severe coronavirus disease 2019 (COVID-19): a meta-analysis. Clin Chim Acta 2020;505:190-1. doi: 10.1016/j.cca.2020.03.004. Epub 2020 Mar 4.

18. Henry BM, Santos de Oliveira MH, Benoit S, Plebani M, Lippi G. Hematologic, biochemical and immune biomarker abnormalities associated with severe illness and mortality in coronavirus disease 2019 (COVID-19): a meta-analysis. Clin Chem Lab Med 2020;58:1021-8.

19. Xu H, Zhong L, Deng J, Peng J, Dan H, Zeng X, et al. High expression of ACE2 receptor of 2019-nCoV on the epithelial cells of oral mucosa. Int J Oral Sci 2020;12:8. 
20. Tan L, Wang Q, Zhang D, Ding J, Huang Q, Tang YQ, et al. Lymphopenia predicts disease severity of COVID-19: a descriptive and predictive study. Signal Transduct Target Ther 2020;5:33.

21. Cervellin G, Schuetz P, Lippi G. Toward a holistic approach for diagnosing sepsis in the emergency department. Adv Clin Chem 2019;92:201-16.

22. Zhou F, Yu T, Du R, Fan G, Liu Y, Liu Z, et al. Clinical course and risk factors for mortality of adult inpatients with COVID-
19 in Wuhan, China: a retrospective cohort study. Lancet 2020;395:1054-62.

23. Chiang $\mathrm{CH}$, Shih JF, Su WJ, Perng RP. Eight-month prospective study of 14 patients with hospital-acquired severe acute respiratory syndrome. Mayo Clin Proc 2004;79:1372-9.

24. Favaloro EJ, Lippi G. Recommendations for minimal laboratory testing panels in patients with COVID-19: potential for prognostic monitoring. Semin Thromb Hemost 2020. doi: 10.1055/s0040-1709498 [Epub ahead of print]. 